\title{
Assessment of psychosocial outcomes in adolescents and young adults with cancer: a systematic review of available instruments
}

This article was published in the following Dove Press journal:

Clinical Oncology in Adolescents and Young Adults

5 February 2013

Number of times this article has been viewed

\author{
Claire E Wakefield ${ }^{1,2}$ \\ Pandora Patterson ${ }^{3}$ \\ Fiona E J McDonald ${ }^{3}$ \\ Helen L Wilson ${ }^{1,2}$ \\ Esther Davis ${ }^{3}$ \\ Ursula M Sansom-Daly ${ }^{2,4}$ \\ 'School of Women's and Children's \\ Health, UNSW Medicine, University \\ of New South Wales, Sydney, NSW, \\ Australia; ${ }^{2}$ Centre for Children's \\ Cancer and Blood Disorders, Sydney \\ Children's Hospital, Sydney, NSW, \\ Australia; ${ }^{3}$ CanTeen, Sydney, NSW, \\ Australia; ${ }^{4}$ School of Psychology, \\ University of New South Wales, \\ Sydney, NSW, Australia
}

Purpose: Given the burgeoning body of research relating to the psychosocial needs of adolescents and young adults (AYAs) with cancer, this review aimed to evaluate the psychometric properties and appropriateness of the instruments available for use in this unique population. Specifically, we reviewed published instruments developed to assess psychological distress (depression, anxiety, stress, and fear of recurrence), psychological growth (resilience, posttraumatic growth, and benefit finding), unmet needs, coping, quality of life, identity, and mindfulness-based practices and skills in AYAs with cancer. Given the dearth of validated instruments targeting AYAs with cancer, this review also provides a summary of promising measures yet to be formally validated in this population.

Methods: Five electronic databases were searched by a team of six researchers, and studies involving AYAs (who have or have had cancer) aged 15-30 years, and published between 1982 and 2012 were reviewed. Of 410 abstracts, 7 instruments were identified as validated in this population, with a further 19 identified as promising.

Results: While there are numerous scales to assess psychosocial outcomes in cancer, few have been specifically validated for AYAs affected by cancer, particularly in the domains of psychological distress, psychological growth, coping, unmet needs, and identity. There are relatively more instruments validated, or promising, for assessment of quality of life than scales for other domains.

Conclusion: In the AYA context, scale selection should be undertaken with thought directed towards the characteristics of this sample (eg, developmental maturity, literacy, and social context), the practicalities of the setting (eg, available funding and resources, time restrictions, and researcher expertise), and the science underlying the scale (eg, theoretical framework and psychometric properties). While multiple measures of psychosocial outcomes are frequently used in AYAs, further research is clearly needed to provide rigorous evidence of the reliability and validity of these tools in young people affected by cancer.

Keywords: adolescents, young adults, psychometric outcomes, psychological outcomes, distress, resilience, coping, quality of life, unmet needs, identity, mindfulness

\section{Introduction}

Increasing research interest in the unique experience of cancer in adolescence and young adulthood has resulted in a burgeoning number of studies investigating psychosocial outcomes in this population. ${ }^{1}$ To date, many of these studies have been descriptive; documenting the prevalence of negative constructs, such as psychological distress. $^{2}$ Others have begun to examine more positive outcomes, such as hopefulness and resilience, ${ }^{3}$ or to investigate the co-occurrence of both negative and positive outcomes within the same study. ${ }^{4,5}$ Interest in the measurement of unmet needs has similarly increased over the past 10 to 20 years. ${ }^{6}$ Evidence indicates that adolescents
Correspondence: Claire Wakefield Centre for Children's Cancer and Blood Disorders, Sydney Children's Hospital, Randwick, NSW 203I, Australia

Tel +6I 293823113

Fax +6I 293821789

Email c.wakefield@unsw.edu.au 
and young adults (AYAs) affected by, or recovering from, cancer are at increased risk of poorer psychological functioning compared to their peers, ${ }^{2}$ or to children and/or adults with cancer. ${ }^{2}$ Consequently, many researchers have turned their attention to developing and evaluating the impact of interventions designed to improve psychosocial outcomes in this population. ${ }^{7-9}$ In this context, the availability of ageappropriate, validated tools to assess psychosocial outcomes is paramount.

AYAs affected by cancer are developmentally unique, meaning that their needs and experiences may not be fully captured by existing instruments developed for children or adults. ${ }^{10}$ Compared to children with cancer, AYAs with cancer may be less likely to comply with their treatment regimen ${ }^{11}$ and are more likely to engage in risky activities during or after their treatment (eg, smoking, unsafe sex).,12 These factors may affect how AYAs transition into well-functioning survivorship. Compared to adults with cancer, AYAs may be less health literate, ${ }^{13}$ have a reduced ability to understand health risks associated with their condition, ${ }^{12}$ and place a relatively higher importance on their peer relationships. ${ }^{14}$ AYAs affected by cancer are also likely to use the Internet and social media more often than children and adults, ${ }^{15}$ are less likely to have adequate insurance, ${ }^{16}$ and are less likely to seek medical attention when confronted with concerning medical symptoms. ${ }^{17,18}$ These issues span across cancer types, age, gender, and cultural groups, and may affect not only the AYA, but also his or her entire family. ${ }^{19}$ Such age-specific individual and social factors are highly likely to shape both the nature and severity of the distress that AYAs with cancer experience. Consequently, existing measures validated for younger children or adults may not be appropriate for the AYA age group.

Conducting research on young people with cancer has specific challenges. These include, but are not limited to: AYAs being less likely to participate in clinical ${ }^{20,21}$ and psychosocial research, ${ }^{22}$ and being less likely to be treated by physicians affiliated with research institutions than other populations. ${ }^{16}$ They are also likely to be spread across both pediatric and adult health care institutions, yet make up a small proportion of the caseload for either type of health care service. ${ }^{16,23}$ They may use different language or jargon than children and adults with cancer, and may find it difficult to communicate with their physician about sensitive issues. ${ }^{24}$ Therefore, as the landscape of AYA research expands, so does the complexity of selecting appropriate measures to assess psychosocial outcomes in this population.
When making decisions about which instruments to use in AYA psychosocial outcomes research, it is important that researchers consider both the potential clinical utility (and appropriateness) of a scale, and its psychometric properties. Relative to the proportion of studies measuring psychosocial outcomes, little research to date has focused on the tools used to perform these measurements. In particular, there is a lack of research identifying and evaluating age-appropriate instruments that are relevant and appealing to young people, comparable across studies, and sensitive to change as AYAs with cancer progress through treatment into survivorship or palliative care.

In this study, therefore, we aimed to review the psychometric properties and appropriateness of the instruments available for use in psychosocial research in AYAs who have, or have had, cancer. Specifically, we reviewed published instruments developed to assess psychological distress (depression, anxiety, stress, and fear of recurrence), psychological growth (resilience, posttraumatic growth, and benefit finding), unmet needs (including readiness for transition to adult health services), coping, quality of life (QOL), and identity in AYAs with cancer. In recognition of the increasing interest in mindfulness-based work with young people, ${ }^{25}$ we also searched for scales assessing mindfulness-based practices and skills in AYAs, such as psychological flexibility, defusion, and expansion. Given the dearth of scales validated for AYAs with cancer, the review also provides a summary of promising instruments that have been used in or developed for AYAs with cancer, but are yet to be formally validated. The key research questions considered were:

1. What validated instruments are available to measure psychosocial outcomes in AYAs who have, or have had, cancer, and what are their psychometric properties?

2. What other promising instruments have been used in or developed for AYAs who have, or have had, cancer that are yet to be formally validated for this population?

3. What are the gaps, if any, in terms of rigorously assessing psychosocial outcomes in young people with cancer?

\section{Methods}

The review was conducted according to gold-standard systematic review procedures. ${ }^{26}$ This involved determining, a priori, a set of inclusion and exclusion criteria; conducting a rigorous literature search across five databases using a standardized abstract screening and study selection process; clearly documenting excluded studies; and using consistent data extraction methods. 


\section{Search strategy}

Five electronic databases were searched; MEDLINE, MEDLINE In Process and Non-Indexed Citations, PsycINFO, EMBASE, and SWABS, and were limited to human studies published in the English language between January 1982 and August 2012. In each database, searches for terms defining the age group, the disease, the study type, the methodology adopted, and the outcomes of interest were run and combined. Specifically, the search algorithm used for all domains was: [AYA OR "emerging adult" OR adolescen\$ OR "young adult" OR teen\$ OR youth] AND [scale OR questionnaire OR measure OR survey] AND [reliability OR validity OR validation OR psychometric] AND [oncol\$ OR neoplasm OR cancer OR tumor OR tumour OR leuk\$] AND limits: [English language] AND [humans] AND [year $=1982$-current]. Domain-specific search terms included: psychological distress [depression OR anxiety OR stress OR fear of recurrence], psychological growth [growth OR resilience OR "benefit finding" OR hope], coping [coping OR cope OR control OR efficacy], QOL [quality of life OR QOL], unmet needs [unmet need\$ OR transition\$], identity [identity] or mindfulness [mindful OR psychological flexibility OR value OR acceptance OR defusion OR expansion].

\section{Inclusion criteria}

Studies published in peer-reviewed journals that validated a measure of one of this study's domains of interest for AYAs were included in this review. Domains reviewed included psychological distress (depression, anxiety, stress, and fear of recurrence), psychological growth (resilience, posttraumatic growth, and benefit finding), unmet needs (including readiness for transition to adult health services), coping (control and efficacy), QOL, identity, and mindfulness-based practices and skills (psychological flexibility, defusion, and expansion) in AYAs with cancer. Excluded from the review were instruments assessing other health-related beliefs (eg, body image, sexuality, spirituality, and fertility concerns), health-related behaviors (eg, sun protection practices, smoking behavior, and physical activity), family functioning (including relationships with parents and siblings), and satisfaction with health care and services. Scales solely assessing symptom-related aspects, such as fatigue, nausea, or pain, were not included, except in cases where these symptom-related aspects formed part of a global assessment of QOL.

In order to capture all possibly relevant instruments in the cancer context and to increase consistency across the field, instruments were included when validated with participants in the same age range, as defined by Clinton-McHarg and colleagues. ${ }^{27}$ The target age range was, therefore, exactly 15-30 years; however, scales developed for individuals younger than 15 years (with an upper age limit of 15-30 years), and scales developed for individuals older than 15 years (but with an upper age limit of 30 years) were also included. Where studies included participants outside this age range (eg, the study only included adults aged 18+), instruments were still included if the study reported subgroup data on any participants within the target age range (eg, if an "adult" study reported data for individuals 18-30 years old separately from the whole dataset).

The age range criteria specifically targeted studies with participants who were currently in the 15-30 age range, regardless of their age at diagnosis of cancer. This approach was adopted in order to identify measurement scales appropriate for patients and other individuals who were currently experiencing the AYA developmental stage. Thus, studies that included survivors of pediatric cancer who were aged 15-30 years were included, while studies assessing outcomes in survivors of adolescent cancer who were older than 30 years when they participated in the research were excluded. Studies were included if participants were receiving active treatment for their illness, were in remission, or had been cured of their condition (ie, were in survivorship). Only the most recent published report of any given instrument was included.

\section{Study selection}

After deduplicating, the resulting 410 abstracts were screened by six researchers in two screening teams (PP, FM, ED and CW, USD, HW) using the inclusion criteria listed above. Inter-rater reliability was $98 \%$ (eight disagreements out of 410 abstracts). Full-text articles were extracted for all abstracts that either appeared eligible to at least one reviewer, or which did not yield sufficient information for review, to confirm eligibility. Consensus between the researchers in each team was achieved by discussing and clarifying details against the full-text article. Studies not fitting the inclusion criteria were discarded, with the primary reasons for exclusion being: (1) lack of data specific to the targeted age range; (2) focus on another domain, such as attitudes toward smoking or safe sexual practices; (3) a medical trial that did not address psychosocial domains; (4) a different primary disease, such as diabetes or HIV; and (5) the instrument was not available in English.

To augment the electronic search, reference lists of included studies and tables of contents of key journals were examined manually to identify additional relevant studies. This led to the inclusion of a further nine articles. 


\section{Assessment of selected instruments}

The team used well-established guidelines ${ }^{28-30}$ and the criteria described in Clinton-McHarg et $\mathrm{al}^{27}$ to evaluate the performance of each instrument. Briefly, each instrument was assessed for seven domains using the following criteria:

1. Internal reliability - acceptable if the reported Cronbach's alpha coefficient was 0.70 or greater. ${ }^{31}$

2. Test-retest reliability - acceptable if the correlations between the measure administered at two time points were $>0.7$ (Pearson or intraclass correlation coefficient) or $>0.6$ (Cohen's kappa co-efficient).

3. Content validity - acceptable if the scale had been evaluated logically by a consensus of expert opinions.

4. Convergent and discriminative validity - acceptable if correlations between similar conceptual constructs were $>0.4$, and/or correlations between conceptually dissimilar constructs were $<0.3$. Alternatively, if factor analysis was conducted, the measure was acceptable if eigenvalues of $>1$ were achieved for each factor.

5. Responsiveness - acceptable if less than $5 \%$ of participants received the highest and lowest scores (ie, floor and/or ceiling effects were minimal) or if the scale was able to detect a change with an effect size of $>0.5$.

6. Acceptability - assessed according to participant burden (ie, number of items and time to complete) and missing items.

\section{Results}

The review identified a total of seven validated instruments assessing psychosocial outcomes in AYAs with cancer (see Tables 1, 3 and 4), and highlighted a further 19 promising scales (see Table 2). The findings from each domain are summarized below.

\section{Measures of psychological distress}

The review identified only one measure, the Uncertainty Scale for Kids, ${ }^{32}$ in the area of depression, anxiety, and stress, and this was available in English only. No specific measures for assessing fear of cancer recurrence were identified.

A number of other measures were identified as promising, namely the Distress Thermometer and the modified Problem List for Adolescents and Young Adults; ${ }^{33,34}$ the Distress Rating Scale (modified version of the Distress Thermometer); ${ }^{35}$ the Beck Youth Inventory-II (BYI-II), ${ }^{36}$ and the Brief Symptom Inventory-18 (BSI-18). ${ }^{36}$ However, the anxiety and depression BYI-II scales are rather lengthy (20 items each) and while the BSI-18 is considerably shorter, it has been developed for individuals 18 years and older. Taking into account that none of these measures have been formally validated in AYAs who have, or have had, cancer, the Distress Thermometer currently appears to be both an accessible and brief option to measure psychological distress in this population.

\section{Measures of psychological growth}

The review identified two measures of psychological growth validated for AYAs with cancer; namely, the Perceived Benefits Scale ${ }^{37}$ and the Herth Hope Index. ${ }^{38}$ Both scales appear to have acceptable internal consistency, are reasonably brief and are available in additional languages (although they have not necessarily been validated for AYAs in these languages). However, neither scale has been assessed for test-retest reliability and are yet to be used in large samples of young people affected by cancer.

One scale was identified as promising - the Benefit/ Burden scale for Children; ${ }^{39}$ however, to date, this has primarily been used for children and younger adolescents with cancer. Other well-known scales, such as the Child and Youth Resilience measure (CYRM-28), ${ }^{40}$ the Child Health and Illness Profile-Adolescent Edition (CHIP-AE) ${ }^{41}$ and the Strengths and Difficulties Questionnaire, ${ }^{42}$ are yet to be validated in AYAs who have, or have had, cancer. Despite strong research interest in posttraumatic growth in cancer, ${ }^{43}$ there was a clear gap in validated scales assessing posttraumatic growth in AYAs with cancer, or in survivors.

\section{Measures of unmet needs}

The review identified one measure of unmet needs developed for, and validated among, the AYA population: the Cancer Needs Questionnaire for Young People (CNQ-YP). ${ }^{44}$ While the CNQ-YP has been validated, it is a relatively new measure and has not been validated in populations outside Australia. Additionally, many of the unmet needs measures developed for the adult population are for specific themes or sub-groups and, hence, can be shorter. No specific instruments addressing transitioning from the pediatric to the adult hospital system met the criteria for promising or validated. Several instruments were found assessing unmet needs in other population groups, in particular with specific adult cancer groups ${ }^{45-47}$ however, none of these had been developed for the AYA population nor had specific data regarding young people affected by cancer. One paper, which investigated satisfaction with information and services provided (which was somewhat related to unmet needs), examined the impact of age and found some differences in their youngest age group of 18-39 year olds, highlighting the need to validate such instruments in the AYA age range. ${ }^{48}$ 


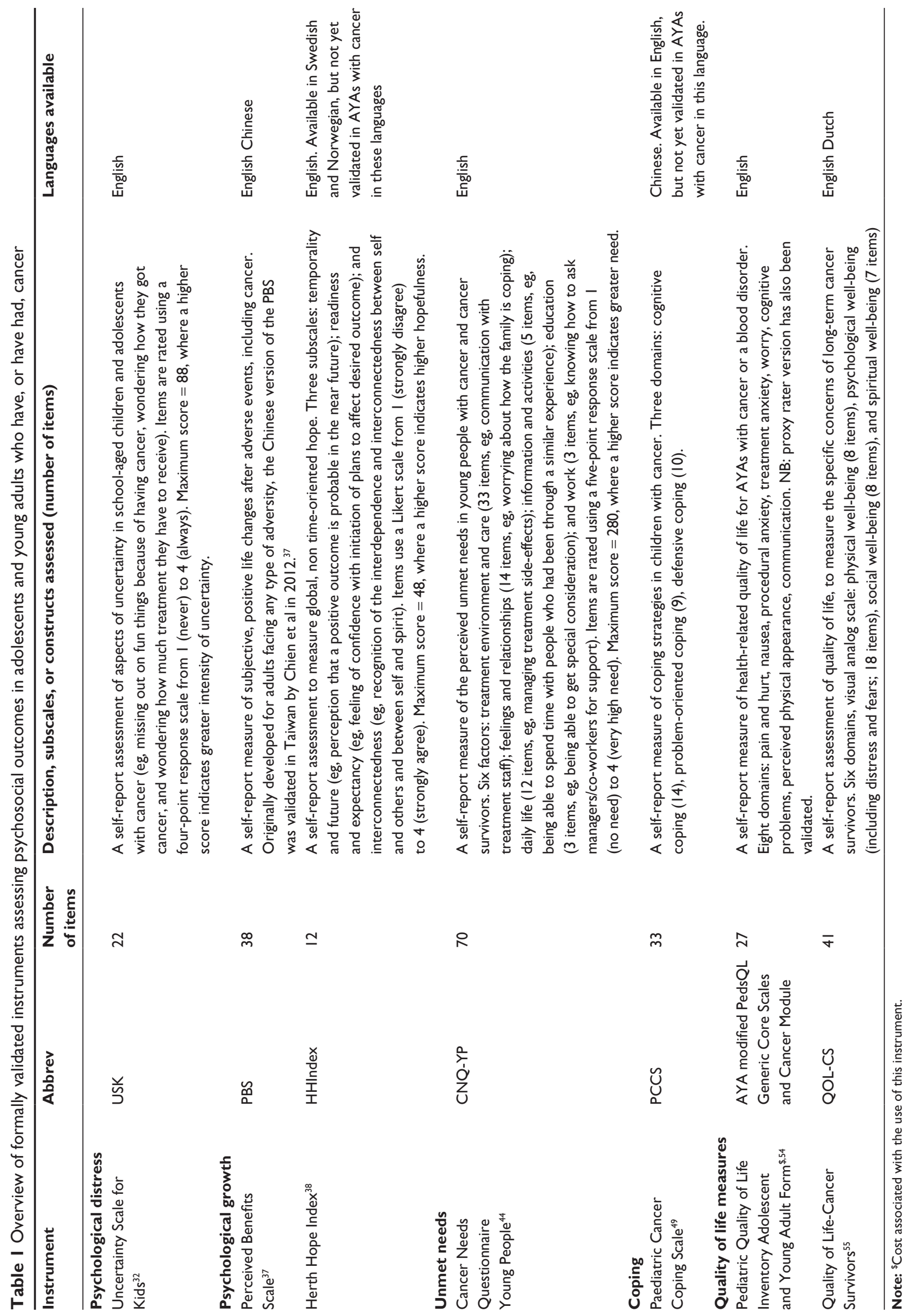




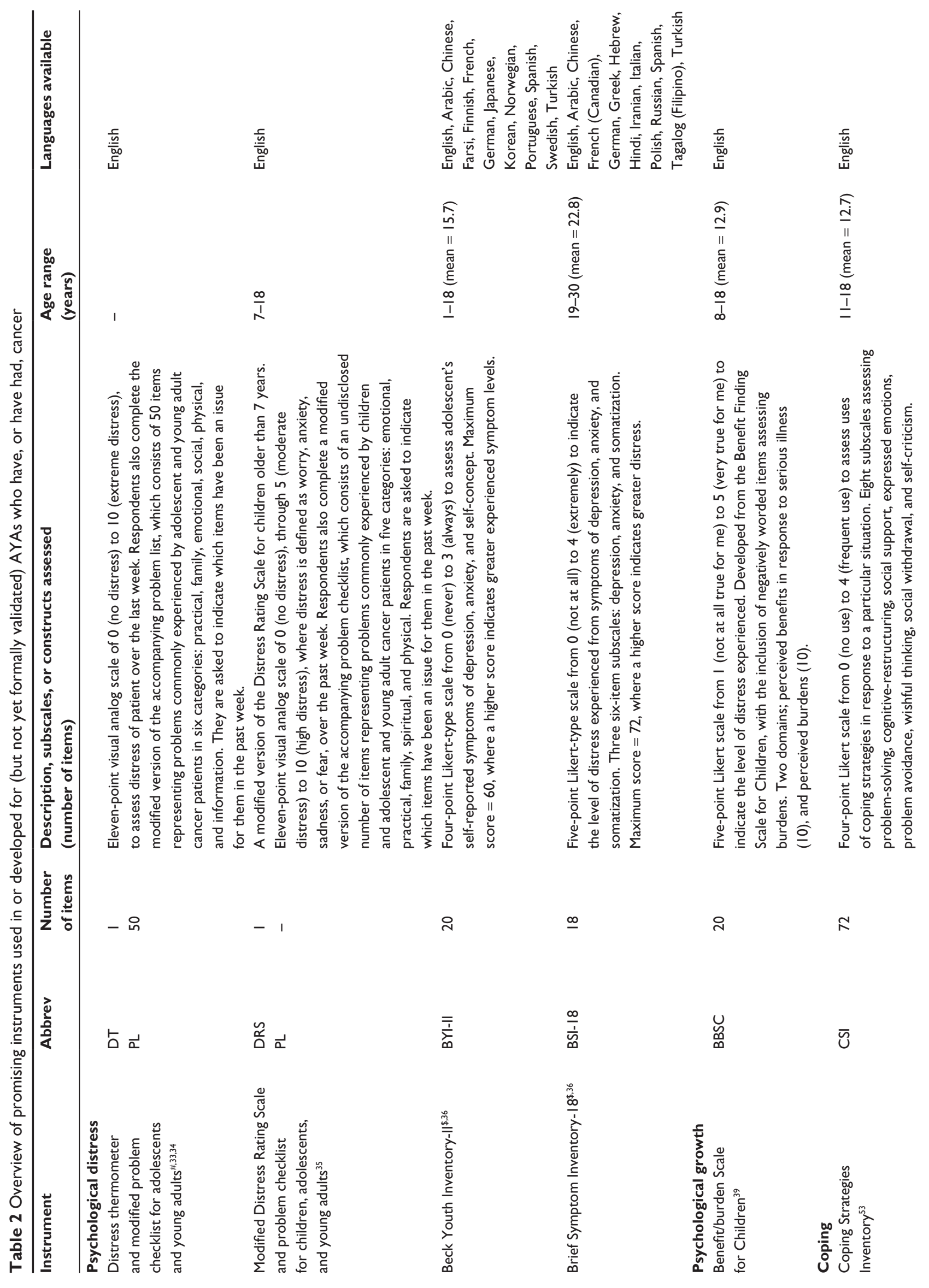




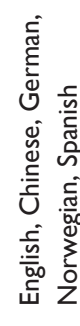

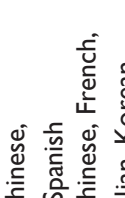

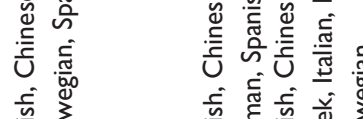

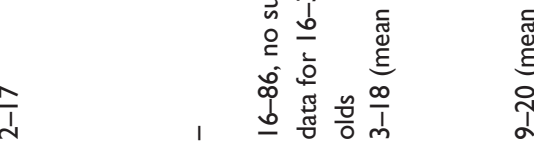

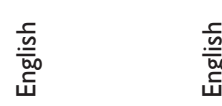

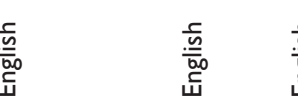

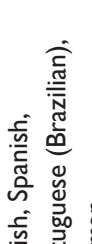

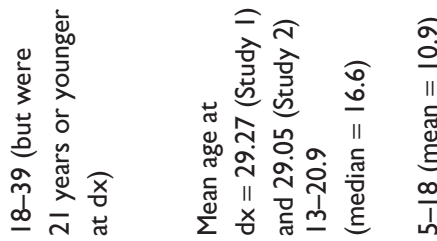

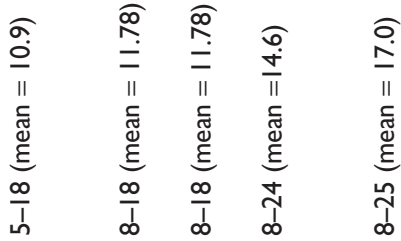

폰

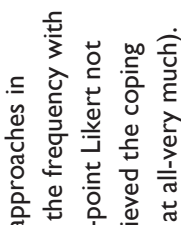

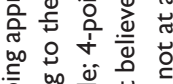

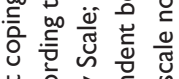

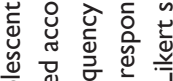

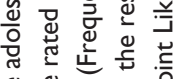

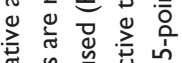

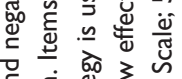

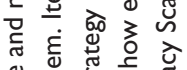

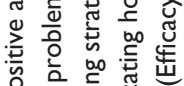

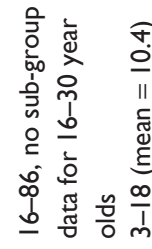

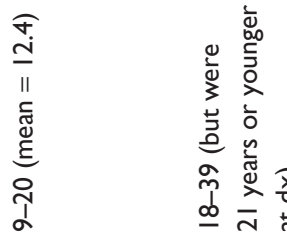

$\therefore \quad$ प्षे

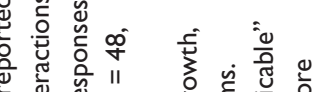

员 论

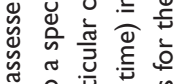

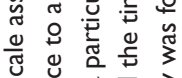

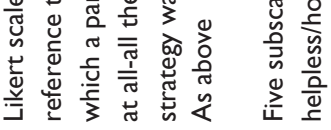

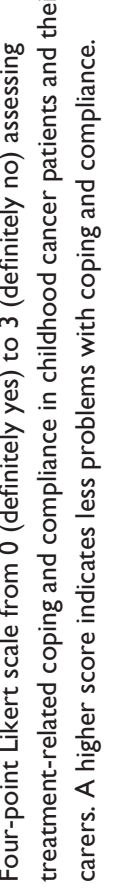

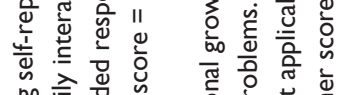

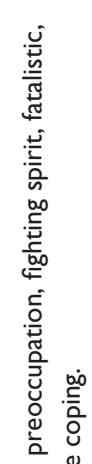

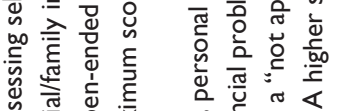

势

ปी

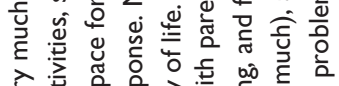

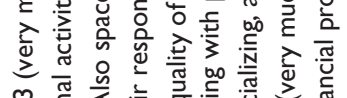

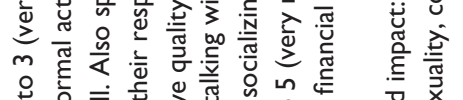

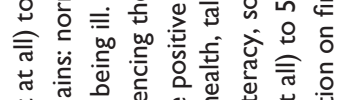

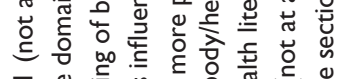

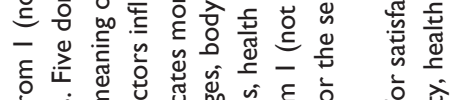

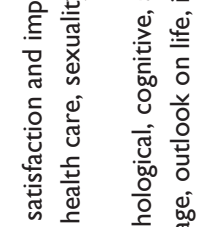

势

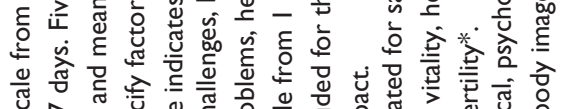

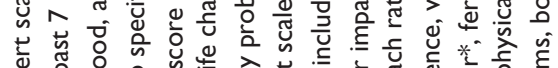

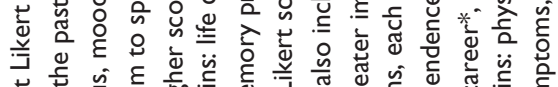

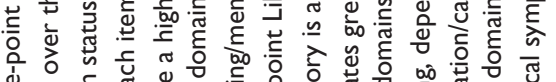

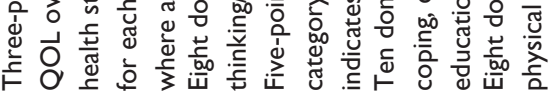

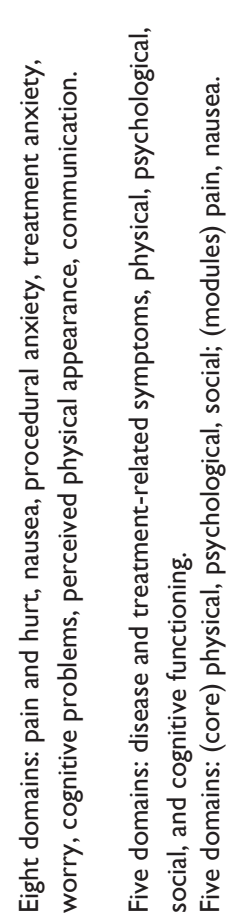

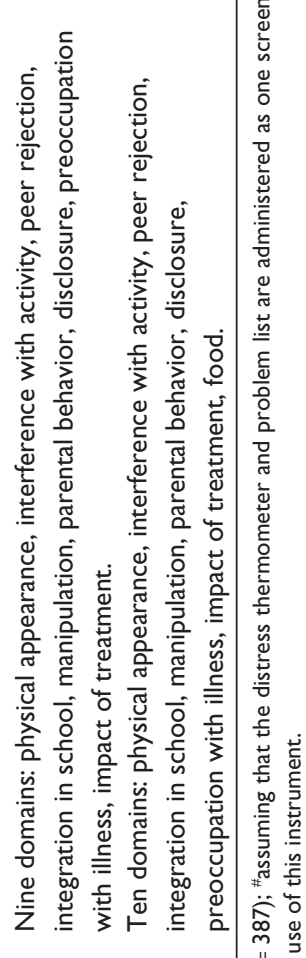

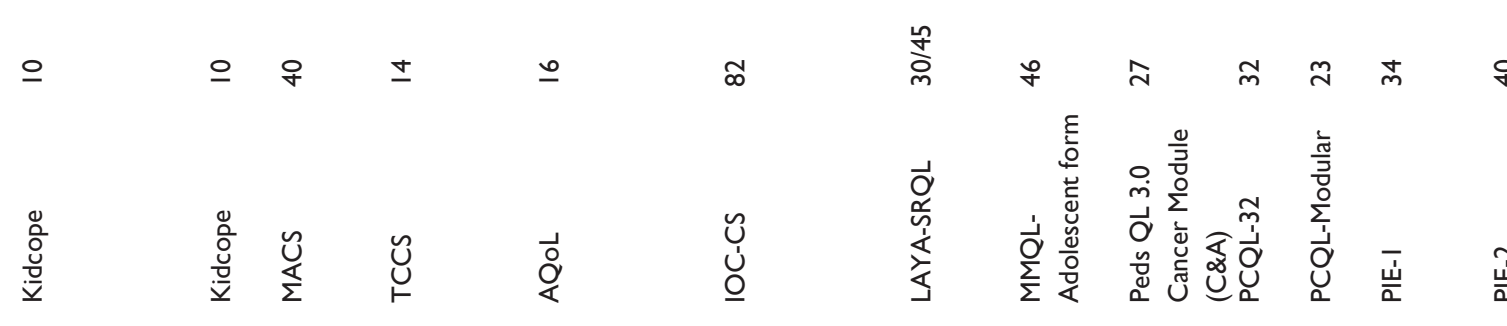

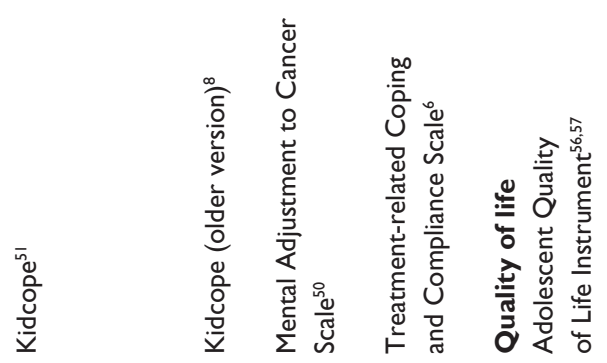

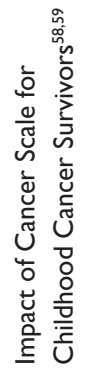

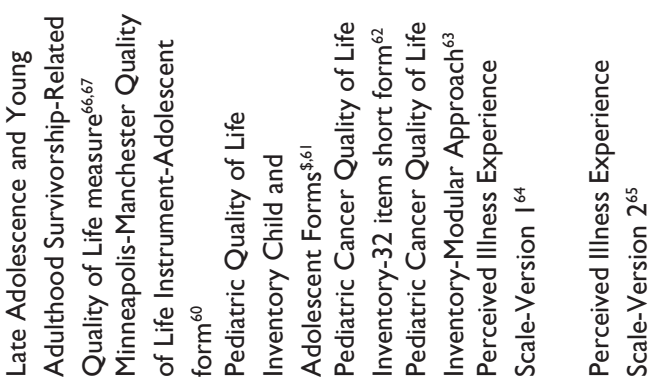

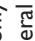

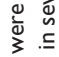

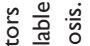

空

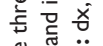

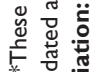




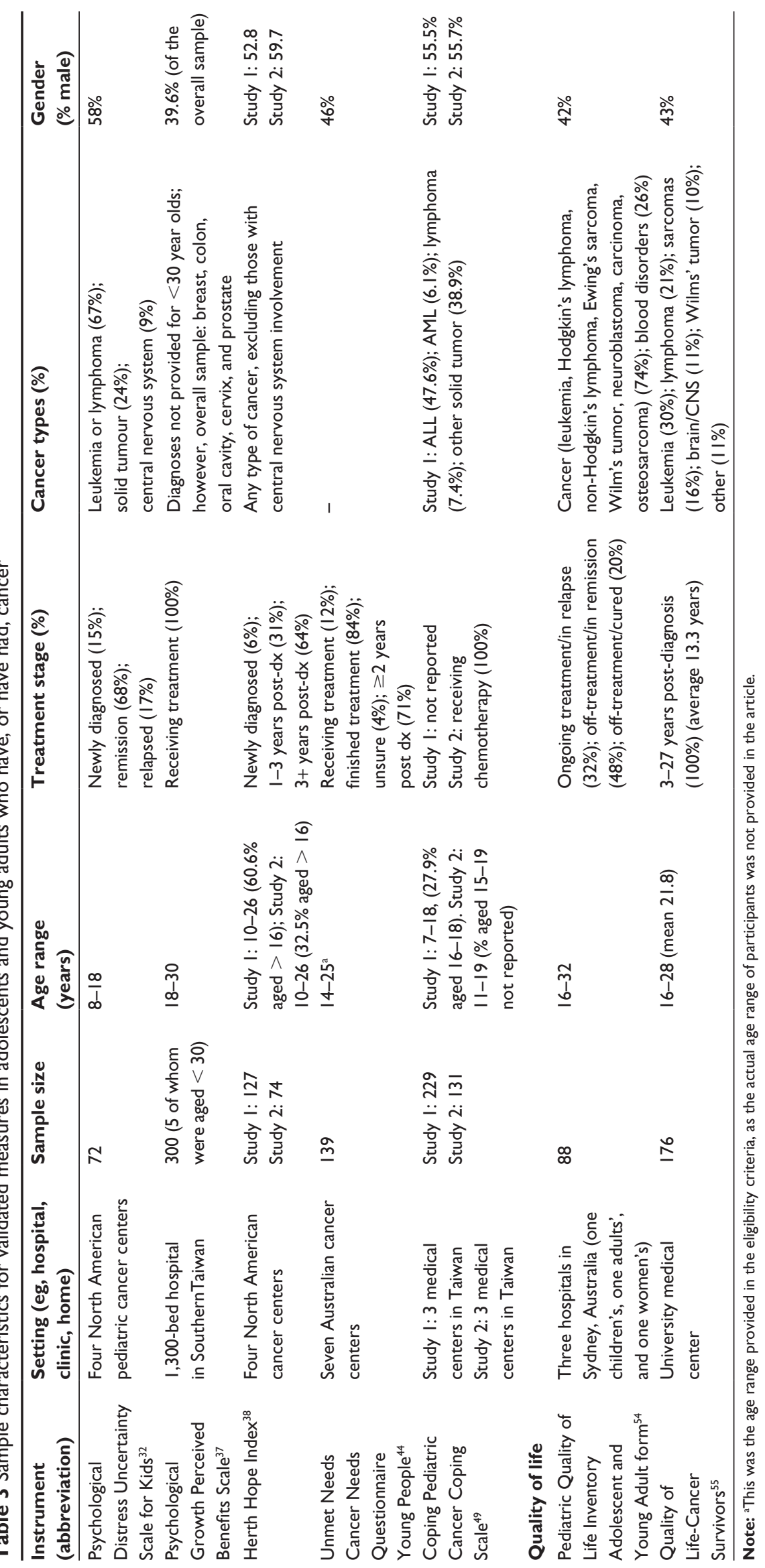




\section{Measures of coping}

One measure of coping validated in the target population was identified, namely, the Pediatric Cancer Coping Scale, which was specifically validated in a Taiwanese AYA population. ${ }^{49}$ While the scale performed well in Taiwanese young people, the English version does not yet appear to have been formally validated in AYAs.

Several instruments were identified as promising coping measures. These include the 14-item Treatment-related Coping and Compliance Scale ${ }^{6}$ and the Mental Adjustment to Cancer Scale, which has been used extensively in cancer, and was administered to cancer patients as young as 16 years old in one study. ${ }^{50}$ The Kid-Cope ${ }^{51}$ has been used in young people with cancer in several studies, ${ }^{8,52}$ and is designed to assess cognitive and behavioral coping strategies. The Coping Strategies Inventory ${ }^{53}$ also showed promise, assessing coping thoughts and behaviors in response to a specific stressful experience.

\section{Measures of QOL}

Of the QOL scales identified, each varied in length (ranging from 14-82 items) and domains measured (five to ten domains per scale). Despite the number of QOL scales available, only two have been validated across the entire age range of AYAs. These include the AYA modified Pediatric Quality of Life Inventory Generic Core Scales (23 items) and Cancer Module (27 items) (PedsQL-AYA ${ }^{54}$ ) and the 41-item Quality of Life-Cancer Survivors scale (QOL-CS). ${ }^{55}$ Both of these scales reported excellent internal consistency, good convergent/discriminant validity, and were able to significantly discriminate between known groups (eg, by symptom severity). These two scales, however, differ in their focus; the QOL-CS is targeted toward childhood cancer survivors, while the PedsQL-AYA incorporates aspects that would be relevant to on- or recently off-treatment AYA cancer patients (eg, nausea and treatment anxiety). Only the PedsQL-AYA reported conducting focus groups with AYA patients, family members, and health or allied health professionals to ensure content validity. Neither scale reported test-retest reliability.

Numerous QOL measures also show promise in the AYA age group. These scales ranged from the very brief Adolescent Quality of Life scale (AQOL, 16 items), ${ }^{56,57}$ to the extensive Impact of Cancer Scale-Childhood Cancer Survivors (IOCS-CS, 82 items), ${ }^{58,59}$ measured between five to ten domains, and have been validated with young people - both those undergoing, and who have finished, cancer treatment. Most of the promising QOL measures were validated for the younger part of the age group (ie, younger adolescents, as young as 8 years old). These included the AQOL,,$^{56,57}$ the Minneapolis-Manchester Quality of Life InstrumentAdolescent form (MMQL-Adolescent) ${ }^{60}$ the Pediatric Quality of Life Inventory-Adolescent form (PedsQL-Adolescent), ${ }^{61}$ which formed the basis of the PedsQL-AYA scale, ${ }^{54}$ the Pediatric Cancer Quality of Life Inventory-32-item short form ${ }^{62}$ and Modular Approach, ${ }^{63}$ and two versions of the Perceived Illness Experience Scale. ${ }^{64,65}$ Two scales have been developed for older AYAs: the IOCS-CS, ${ }^{58,59}$ and the Late Adolescent and Young Adult - Survivorship-Related Quality of Life measure (LAYA-SRQL). ${ }^{66,67}$

All promising measures appeared to have acceptable internal consistency, and several were able to significantly detect differences between young people according to treatment stage (AQOL, MMQL-Adolescent, PCQL-32, PCQLModular, and PIE). However, few of these scales reported test-retest reliability or sensitivity to change over time.

\section{Measures of identity}

The review did not identify any scales to assess the construct of identity, nor were there any suitable for the promising classification. This finding represents a clear gap given both the importance of this construct for the AYA population and the potential impact of a life-threatening disease, such as cancer, on identity development. ${ }^{14,68}$

\section{Measures of mindfulness-based practice and skills}

As with the domain of identity, no validated mindfulnessbased measures were identified from the review and there were none that fit the promising classification. Given the growing body of literature attesting to the benefits of mindfulness-based practices and skills within the cancer population, ${ }^{69-71}$ including AYAs, ${ }^{25}$ it would seem pertinent to have access to scales validated with AYAs with cancer measuring this construct.

\section{Discussion}

The ways in which clinicians, psychologists and researchers think about and define outcomes in young people are varied. The present paper evolved out of our own and our colleagues' recognition of the difficulties encountered when selecting the most suitable or appropriate outcome measures for conducting research in this age group. The review highlights the dearth of instruments available to assess psychosocial outcomes in young people affected by cancer, and reveals an interesting imbalance of available instruments across domains. While some domains are understandably under-represented, given their relatively 
Table 4 Psychometric properties of included instruments

\begin{tabular}{llll}
\hline $\begin{array}{l}\text { Instrument } \\
\text { (abbreviation) }\end{array}$ & Internal consistency ${ }^{\mathrm{a}}$ & $\begin{array}{l}\text { Test-retest reliability, } \\
\text { time period }^{\mathrm{b}}\end{array}$ & Content validity \\
\hline
\end{tabular}

\section{Psychological distress}

USK ${ }^{32}$

0.94

$r=0.64, P=0.005$ (I week)

Psychological growth

$\mathrm{PBS}^{37}$

Positive attitude (0.98);

positive belief $(0.94)$

HHIndex ${ }^{38}$

Study I (0.84);

Study 2 (0.78)

Unmet needs

CNQ-YP ${ }^{44}$

Treatment environment and care (0.98);

feelings and relationships (0.92); daily

life (0.94); information and activities

$(0.83)$; education $(0.82)$; work $(0.89)$

Coping

PCCS $^{49}$

Study I : 0.91

Study I: $0.86(2$ weeks, $n=45)$

NA

Not tested because the stability of hope is not well demonstrated in cancer populations and patient access limited

Weighted kappa ranged from 0.09 to 0.94 , with all but four items reaching weighted kappa values above 0.60

Two panels assessed content validity; one of children and adolescents with cancer, the other of clinical nurse experts. For 20 of the 22 original items, the majority of experts agreed that the item was "good" or "okay." The remaining 2 items were reworded substantially using children's comments.

Rasch analysis demonstrated that, of the 38 items in the PBS, 28 items were applicable to cancer patients, assessing two distinct domains: positive attitude and positive belief. Use of the HHIndex based on the Adolescent Resilience Model.

The model was developed via a series of qualitative and quantitative studies in AYAs with chronic illnesses, including cancer.

NA

\begin{abstract}
Qualitative interviews about coping experiences of young people with cancer were used to develop the PCCS, also compared with existing measures. Content validity assessed by expert panel. Face validity assessed with child patients as well.
\end{abstract}

Quality of life

AYA modified PedsQL Generic Core Scales: Five of five domains NA

Generic Core Scales $\geq 0.70$. Total score: 0.95; physical health

and Cancer Module ${ }^{54}$ (0.93); psychosocial health (0.92);

emotional functioning $(0.86)$; social

functioning $(0.8 \mathrm{I})$; study/work functioning

(0.88) Cancer Module: Eight of eight

domains $\geq 0.70$; Pain/hurt (0.75); nausea

(0.89); procedural anxiety $(0.85)$; treatment anxiety (0.83); worry (0.76); cognitive

problems $(0.90)$; perceived physical

appearance (0.77); communication (0.76)

QOL-CS 55

Total scale (0.87); physical $(0.8 \mathrm{I})$;

psychological (0.82); fears (0.88);

NA

Adapted from AYA modified PedsQL

Generic Core Scales and Cancer Module.

Two focus groups conducted: Ist $(n=12)$

AYA patients and family members. 2nd

$(n=8)$ health and allied health professionals (nurses, doctors, social workers caring for AYA patients). Wording modifications approved by PedsQL principal developer and copyright holder.

NA social $(0.76)$; spiritual $(0.78)$

Notes: aCronbach's alpha (total scale, subscales). Coefficients reported here are derived either from the AYA population or from the original published validation study of each scale. Many Cronbach's coefficients have later been calculated using scales in different populations, and these provide valuable information about the psychometric properties of the scale in different contexts; however, these are not reported here; bintraclass correlation.

Abbreviations: AYA, adolescent and young adults; NA, not assessed or reported.

recent investigation (eg, mindfulness-based practice) ${ }^{69}$ other domains are already being assessed regularly, yet remain poorly tested in AYAs with cancer (eg, psychological distress, specifically anxiety and depression). ${ }^{7}$ In contrast, QOL instruments are relatively well-tested in this population. ${ }^{27}$
Despite the plethora of research into cancer and psychological distress, ${ }^{72}$ the endorsement of distress being the sixth vital sign of cancer, ${ }^{73}$ the acknowledgement of the prevalence of distress amongst young cancer patients at all stages of illness, ${ }^{19}$ and the growing practice of cancer 
Anxiety $(r=0.56, P=0.001)$

Depressive symptoms $(r=0.59, P<0.00 \mathrm{I})$

Cancer knowledge $(r=-0.27, P=0.02)$

Analysis of variance on the two subscales demonstrated that duration of support group attendance was related to PBS score, such that the longer the patient had attended a support group, the more positive changes they reported

Moderate convergent correlations with resilience (self-esteem $[r=0.62]$, self-confidence [0.57], self-transcendence [0.58]) and QOL [0.33]. Mod-low discriminant correlations with uncertainty in illness [-0.42] and symptom distress [-0.22]. Factor analysis supported one-factor solution, final model: $\mathrm{NNFI}=0.97$, $\mathrm{CFI}=0.98 ; \mathrm{IFI}=0.98 ; \mathrm{RMSEA}=0.04$

Measure could not discriminate between participants receiving treatment and those who had finished treatment $(P<0.05)$

Study I: Construct validity established with $\mathrm{KMO}=0.87$, three factors identified with eigenvalues $>$ I (cognitive coping, problem-oriented coping, defensive coping). Cognitive coping and problem-oriented coping correlated with resilience $(r=0.49,0.6 \mathrm{I})$. Defensive coping correlated with anxiety $(0.47)$, worry $(0.5 \mathrm{I})$, physiological $(0.30)$ and social $(0.37)$ anxiety

Six-factors achieved Eigenvalues $>1.0$, (76\% of variance).

Convergent validity with Generic Core Scale: $31 / 48$ correlations $>0.4$ in expected direction
Highest mean (SD) for item is 2.77 (I.2)

Lowest mean (SD) for item is 1.65 (I.0)
NA

NA

Mean scores (SD) for patients $<30$ :

Positive attitude $=0.9(0.44)$;

positive belief $=0.92(0.35)$

NA

The original 30 -item scale was reduced to 12 items to reduce participant burden on AYAs with cancer

Proportion who scored the minimum score for each factor ranged from $8.3 \%$ to $43 \%$; for maximum score: 0-5.I (large proportion of participants had floor effects in education (42\%) and work (43\%))

Proportion of participants who scored the minimum scores for each item ranged from $2 \%-36.8 \%$; for the maximum score: II. $9 \%-68.2 \%$

Significantly discriminates between AYAs who report mild, moderate, and severe symptom severity across all eight subscales. Eight of eight subscales showed $\geq 8 \%$ floor + ceiling effects combined (range: $8 \%-70 \%$ )
Easy to follow (80\%); clear (73\%); easy to understand (56\%); not distressing (78\%)

NA

Minimal missing data $(\leq 10 \%)$
Cancer Specific Worry Scale; Psychosocial Worry Scale; General Health Worry Scale.

Nine correlations $>0.40$
Significantly discriminates between participants' medical history, cancer after-effects, gender, income, marital status $(P<0.05$ for $5 / 6$ factors) programs screening for distress in a standardized manner, ${ }^{74}$ there are few distress-related measures that have been formally validated in AYAs with cancer. There are also clear gaps in the availability of validated unmet needs, coping, and psychological growth instruments for AYAs with cancer.
The development of instruments measuring satisfaction with services provided (including psychosocial services) may represent the next stage beyond unmet needs measures and deserves further exploration. Also striking is the absence of measures examining needs or knowledge around the complex 
issue of transitioning from pediatric to adult care, despite clinical and research acknowledgement that transition is a key time of need for young people with cancer. ${ }^{75,76}$

In contrast, the development of QOL measures for AYAs with, or recovering from, cancer is more advanced (particularly for younger adolescents). While many of these scales incorporate assessment of young peoples' emotional wellbeing and/or functioning, QOL measures are a more distal measure of distress than instruments targeting specific psychological domains. This is interesting, given that many interventions have been developed to target specific psychological domains (eg, depression, anxiety, and posttraumatic stress), yet many use QOL as their primary outcome measure given the lack of other validated alternatives. QOL instruments typically comprise a mixture of variables that may cause poor QOL ("causal variables," eg, nausea) and variables that may be reflective of poor QOL in general ("indicator variables," eg, distress), ${ }^{77}$ and so it is possible that psychosocial interventions may be able to change the indicator variables (eg, reduce distress) but not the causal variables, as these variables are more difficult to change and do not necessarily belong to the same latent construct (eg, nausea, communication issues, and financial concerns).${ }^{77}$ Consequently, global QOL assessment comprising a mix of related factors may form a less sensitive measure of distress or emotional wellbeing than other more specific constructs.

In addition, neither of the two QOL scales validated across the AYA age range (PedsQL-AYA and the QOL-CS), appear to comprehensively assess AYA-specific issues, such as drug/alcohol consumption, fertility, sexuality/intimacy, or dating concerns (although there are some scales specifically assessing these aspects of the young person's experience not captured here). By contrast, the two promising QOL scales identified for older AYAs (IOCS-CS and LAYA-SRQL), do include aspects that may be important for the whole AYA age range (eg, body image, financial issues, career, and sexuality and fertility concerns). These content aspects may make these scales uniquely applicable to the developmental concerns of AYAs, unlike many of the instruments developed for younger adolescents.

\section{Implications for future instrument development}

Finding instruments tested across the entire AYA age range is challenging. Both pediatric and adult cancer fields, therefore, need to be aware of the unique psychosocial issues of AYAs and consider the needs of this population when developing pediatric and adult instruments. One solution may be for the age groups of potentially useful scales to be extended (upwards or downwards) to capture an age span between 15 and 25 years. Even without extending the age ranges tested for existing instruments, presenting validation data for subgroups of AYAs separately would be beneficial in order to assess whether a general scale is valid and reliable in this specific age range (eg, presenting subgroup data for 15-18 year olds completing children's scales, or for 18-25 year olds completing adult scales, separately). This would ensure that scales covering more domains could be used in AYAs and may also contribute to reducing the "gap" between AYA and adult outcomes ${ }^{23,78}$ by ensuring that their psychosocial functioning can be reliably and validly assessed.

There is also clear need for instruments already commonly used in young people with cancer (despite not having been validated in this population) to be tested to ensure the unique needs of young people are captured by these instruments (eg, the Distress Thermometer and modified Problem List for Adolescents and Young Adults; ${ }^{34}$ Hospital Anxiety and Depression Scale $;{ }^{79}$ the Depression, Anxiety and Stress Scale $;^{80}$ and the K-10 ${ }^{81}$ ). Across the pool of scales identified in all psychosocial domains, there was also a lack of consistent reporting of important validity data such as test-retest reliability, responsiveness/sensitivity to change over time, and acceptability/relevance of the final scale to consumers representing different groups (eg, young people who are on versus off cancer treatment).

Consideration should also be given to validating existing measures specifically developed for young people with the AYA cancer population. For example, mindfulness-based practice and skills is an area of increasing interest and existing measures, such as the Child and Adolescent Mindfulness Measure (CAMM) ${ }^{82}$ and the Avoidance and Fusion Questionnaire for Youth (AFQ-Y) ${ }^{83}$ could be very useful in measuring these important constructs in young people who have, or have had, cancer.

Finally, tools specifically developed to assess the unique experience of coping with cancer, psychological growth (eg, resilience), and identity in AYAs with cancer are also urgently needed. So too are instruments developed for specific needs groups, including AYAs with culturally and linguistically diverse backgrounds (recognized as a critical variable in families of children with cancer, ${ }^{84}$ adults with cancer, ${ }^{85,86}$ and in adolescents with chronic illness ${ }^{87}$ ), as well as other minority groups (eg, individuals with same-sex attraction $\left.{ }^{88}\right)$. 


\section{Implications for future instrument selection}

It is unclear how often researchers are using the "best available" instrument to assess the impact of cancer and/or its treatment on young people. Researchers may also not be using valid and reliable instruments when assessing the efficacy of psychosocial or other interventions developed for this age group. Future instrument selection, therefore, must be undertaken with care and thought directed toward the characteristics of the AYA sample (eg, literacy, culture, medical condition), the practicalities of the setting (eg, available funding and resources, time restrictions, and researcher expertise), the specific aspect of the cancer experience to be studied, and the science underlying the scale (eg, theoretical framework and psychometric properties). Administratively, research instruments need to be easy for clinic staff to distribute, and should aim to minimize the burden on young respondents.

\section{Conclusion}

The goal of this review was to provide clinicians and researchers with concise and workable descriptions of all the published instruments assessing psychosocial functioning in the AYA oncology setting. Recent research has contributed a great deal to our understanding of AYAs' experiences when being treated for, ${ }^{5}$ or recovering from, ${ }^{89}$ cancer. However, a broader investigation of the complexities of AYA outcomes and their measurement is clearly warranted. These findings have important implications for how future interventions for this population will be developed and evaluated. The limited number of available tools in psychosocial domains (other than QOL) may be seriously restricting researchers' abilities to validly and sensitively measure impact, or change, in psychosocial functioning in an important cohort of individuals affected by cancer.

\section{Acknowledgments}

Dr Claire Wakefield is supported by an Early Career Development Award from the Cancer Institute of NSW (ID 11/ ECF/3-43). The Behavioural Sciences Unit at Sydney Children's Hospital is supported by the Kids with Cancer Foundation. Ms Ursula Sansom-Daly is supported by a Leukaemia Foundation of Australia PhD Scholarship.

\section{Disclosure}

The authors report no conflicts of interest in this work.

\section{References}

1. Thomas DM, Albritton KH, Ferrari A. Adolescent and young adult oncology: an emerging field. J Clin Oncol. 2010;28(32):4781-4782.
2. Seitz D, Besier T, Debatin KM, et al. Posttraumatic stress, depression and anxiety among adult long-term survivors of cancer in adolescence. Eur J Cancer. 2010;46(9):1596-1606.

3. Juvakka T, Kylmä J. Hope in adolescents with cancer. Eur J Oncol Nurs. 2009;13(3):193-199.

4. Haase JE, Phillips CR. The adolescent/young adult experience. $J$ Ped Onc Nurs. 2004;21(3):145-149.

5. Bellizzi KM, Smith A, Schmidt S, et al; Adolescent and Young Adult Health Outcomes and Patient Experience (AYA HOPE) Study Collaborative Group. Positive and negative psychosocial impact of being diagnosed with cancer as an adolescent or young adult. Cancer. 2012;118(20):5155-5162.

6. Felder-Puig R, Gallo A, Waldenmair M, Gadner H, Topf R. The TCCSa short measure to evaluate treatment-related coping and compliance in hospitalised childhood cancer patients and their primary caregivers. Support Care Cancer. 2004;12(1):41-47.

7. Seitz DC, Besier T, Goldbeck L. Psychosocial interventions for adolescent cancer patients: a systematic review of the literature. Psychooncology. 2009;18(7):683-690.

8. Sansom-Daly UM, Wakefield CE, Bryant RA, et al. Online group-based cognitive-behavioural therapy for adolescents and young adults after cancer treatment: A multicenter randomised controlled trial of Recapture Life-AYA. BMC Cancer. 2012;12(1):339.

9. Kazak AE, Simms S, Barakat L, et al. Surviving cancer competently intervention program (SCCIP): a cognitive-behavioral and family therapy intervention for adolescent survivors of childhood cancer and their families. Fam Process. 1999;38(2):175-191.

10. Tonorezos ES, Oeffinger KC. Research challenges in adolescent and young adult cancer survivor research. Cancer. 2011;117(S10):2295-2300.

11. Butow P, Palmer S, Pai A, Goodenough B, Luckett T, King M. Review of adherence-related issues in adolescents and young adults with cancer. J Clin Oncol. 2010;28(32):4800-4809.

12. Morgan S, Davies S, Palmer S, Plaster M. Sex, drugs, and rock 'n' roll: caring for adolescents and young adults with cancer. J Clin Oncol. 2010;28(32):4825-4830.

13. Nagel K, Wizowski L, Duckworth J, Cassano J, Hahn SA, Neal M. Using plain language skills to create an educational brochure about sperm banking for adolescent and young adult males with cancer. J Ped Onc Nurs. 2008;25(4):220-226.

14. Jones BL, Parker-Raley J, Barczyk A. Adolescent cancer survivors: identity paradox and the need to belong. Qual Health Res. 2011;21(8): 1033-1040.

15. Schiffman JD, Csongradi E, Suzuki LK. Internet use among adolescent and young adults (AYA) with cancer. Pediatr Blood Cancer. 2008; 51(3):410-415.

16. Burke ME, Albritton K, Marina N. Challenges in the recruitment of adolescents and young adults to cancer clinical trials. Cancer. 2007; 110(11):2385-2393.

17. Martin S, Ulrich C, Munsell M, Taylor S, Lange G, Bleyer A. Delays in cancer diagnosis in underinsured young adults and older adolescents. Oncologist. 2007;12(7):816-824.

18. Wakefield CE, McLoone JK, Fleming CAK, et al. Adolescent cancer and health-related decision-making: An Australian multi-perspective family analysis of appointment attendance and involvement in medical and lifestyle choices. J Adolesc Young Adult Oncol. 2012;1(4): $173-180$.

19. Zebrack B, Isaacson S. Psychosocial care of adolescent and young adult patients with cancer and survivors. J Clin Oncol. 2012;30(11): 1221-1226.

20. Ferrari A, Montello M, Budd T, Bleyer A. The challenges of clinical trials for adolescents and young adults with cancer. Pediatr Blood Cancer. 2008;50(S5):1101-1104.

21. Sender LS. Approaches to increasing clinical trial access and enrollment for adolescents and young adults with cancer. J Adolesc Young Adult Oncol. 2011;1(2):69. 
22. Aubin S. Challenges conducting qualitative psychosocial research for adolescent and young adult patients and survivors. J Adolesc Young Adult Oncol. 2011;1(2):71-76.

23. Ferrari A, Thomas D, Franklin ARK, et al. Starting an adolescent and young adult program: Some success stories and some obstacles to overcome. J Clin Oncol. 2010;28(32):4850-4857.

24. Quinn GP, Vadaparampil ST. Fertility preservation and adolescent/ young adult cancer patients: Physician communication challenges. J Adolesc Health. 2009;44(4):394-400.

25. Burke CA. Mindfulness-based approaches with children and adolescents: A preliminary review of current research in an emergent field. J Child Fam Stud. 2010;19(2):133-144.

26. Mokkink LB, Terwee CB, Stratford PW, et al. Evaluation of the methodological quality of systematic reviews of health status measurement instruments. Qual Life Res. 2009;18(3):313-333.

27. Clinton-McHarg T, Carey M, Sanson-Fisher R, Shakeshaft A, Rainbird K. Measuring the psychosocial health of adolescent and young adult (AYA) cancer survivors: a critical review. Health Qual Life Outcomes. 2010;8(1):25.

28. Bryman A, Cramer D. Quantitative Data Analysis for Social Scientists. Revised ed. London: Routledge; 1994.

29. McDowell I, Newell C. Measuring Health: A Guide to Rating Scales and Questionnaires. 2nd ed. New York: Oxford University Press; 1996.

30. Streiner D, Norman G. Health Measurement Scales. Oxford: Oxford University Press; 1995.

31. Nunnally J. Introduction to Psychological Measurement. New York: McGraw-Hill; 1970.

32. Stewart JL, Lynn MR, Mishel MH. Psychometric evaluation of a new instrument to measure uncertainty in children and adolescents with cancer. Nurs Res. 2010;59(2):119-126.

33. CanTeen. Adolescent and Young Adult Oncology Psychosocial Care Manual. Australia: CanTeen; 2011.

34. Palmer S, Patterson P, Thompson K. A national approach to improving adolescent and young adult (AYA) oncology psychosocial care: the development of AYA-specific psychosocial assessment and care tools. Palliat Support Care. 2012. In press.

35. Patel SK, Mullins W, Turk A, Dekel N, Kinjo C, Sato JK. Distress screening, rater agreement, and services in pediatric oncology. Psychooncology. 2011;20(12):1324-1333.

36. Liptak C, Manley P, Recklitis C. The feasibility of a psychosocial screening program for survivors of pediatric brain tumors. Neuro Oncol. 2012;14:i136.

37. Chien TW, Wang WC, Chien CC, Hwang WS. Rasch analysis of positive changes following adversity in cancer patients attending community support groups. Psychooncology. 2011;20(1):98-105.

38. Phillips-Salimi CR, Haase JE, Kintner EK, Monahan PO, Azzouz F. Psychometric properties of the Herth Hope Index in adolescents and young adults with cancer. J Nurs Meas. 2007;15(1): $3-23$.

39. Currier JM, Hermes S, Phipps S. Brief report: Children's response to serious illness: Perceptions of benefit and burden in a pediatric cancer population. J Pediatr Psychol. 2009;34(10):1129-1134.

40. Liebenberg L, Ungar M, Van de Vijver F. Validation of the child and youth resilience measure-28 (CYRM-28) among Canadian youth. Res Soc Work Pract. 2012;22(2):219-226.

41. Starfield B, Ensminger M, Riley A, et al. Adolescent health status measurement: development of the Child Health and Illness Profile. Pediatrics. 1993;91(2):430-435.

42. Goodman R. Psychometric properties of the strengths and difficulties questionnaire. J Am Acad Child Adolesc Psychiatry. 2001;40(11): 1337-1345.

43. Barakat LP, Alderfer MA, Kazak AE. Posttraumatic growth in adolescent survivors of cancer and their mothers and fathers. $J$ Pediatr Psychol. 2006;31(4):413-419.
44. Clinton-McHarg T, Carey M, Sanson-Fisher R, D'Este C, Shakeshaft A. Preliminary development and psychometric evaluation of an unmet needs measure for adolescents and young adults with cancer: the Cancer Needs Questionnaire - Young People (CNQ-YP). Health Qual Life Outcomes. 2012;10:13.

45. Boyes A, Girgis A, Lecathelinais C. Brief assessment of adult cancer patients' perceived needs: development and validation of the 34-item Supportive Care Needs Survey (SCNS-SF34). J Eval Clin Pract. 2009;15(4):602-606.

46. Cossich T, Schofield P, McLachlan S. Validation of the cancer needs questionnaire (CNQ) short-form version in an ambulatory cancer setting. Qual Life Res. 2004;13(7):1225-1233.

47. Coyle N, Goldstein ML, Passik S, Fishman B, Portenoy R. Development and validation of a patient needs assessment tool (PNAT) for oncology clinicians. Cancer Nurs. 1996;19(2):81.

48. Ross L, Petersen MA, Johnsen AT, Lundstrøm LH, Groenvold M. Are different groups of cancer patients offered rehabilitation to the same extent? A report from the population-based study "The Cancer Patient's World”. Support Care Cancer. 2012;20(5):1089-1100.

49. Wu LM, Chin CC, Chen CH, Lai FC, Tseng YY. Development and validation of the paediatric cancer coping scale. JAdv Nurs. 2011;67(5): 1142-1151.

50. Schwartz CE, Daltroy LH, Brandt U, Friedman R, Stolbach L. A psychometric analysis of the Mental Adjustment to Cancer scale. Psychol Med. 1992;22(01):203-210.

51. Spirito A, Stark LJ, Williams C. Development of a brief coping checklist for use with pediatric populations. J Pediatr Psychol. 1988;13(4): 555-574.

52. Brown RT, Kaslow NJ, Hazzard AP, et al. Psychiatric and family functioning in children with leukemia and their parents. $J$ Am Acad Child Adolesc Psychiatry. 1992;31(3):495-502.

53. Trask PC, Paterson AG, Trask CL, Bares CB, Birt J, Maan C. Parent and adolescent adjustment to pediatric cancer: associations with coping, social support, and family function. J Ped Onc Nurs. 2003; 20(1):36-47.

54. Ewing JE, King MT, Smith NF. Validation of modified forms of the PedsQL generic core scales and cancer module scales for adolescents and young adults (AYA) with cancer or a blood disorder. Qual Life Res. 2009;18(2):231-244.

55. Zebrack B, Chesler M. A psychometric analysis of the Quality of LifeCancer Survivors (QOL-CS) in survivors of childhood cancer. Qual. Life Res. 2001;10(4):319-329.

56. Ward-Smith P, Hamlin J, Bartholomew J, Stegenga K. Quality of life among adolescents with cancer. J Ped Onc Nurs. 2007;24(3): $166-171$.

57. Ward-Smith P, McCaskie B, Rhoton S. Adolescent-evaluated quality of life: a longitudinal study. J Ped Onc Nurs. 2007;24(6): 329-333.

58. Zebrack BJ, Ganz PA, Bernaards CA, Petersen L, Abraham L. Assessing the impact of cancer: development of a new instrument for long-term survivors. Psychooncology. 2006;15(5):407-421.

59. Zebrack BJ, Donohue JE, Gurney JG, Chesler MA, Bhatia S, Landier W. Psychometric evaluation of the Impact of Cancer (IOC-CS) scale for young adult survivors of childhood cancer. Qual Life Res. 2010;19(2):207-218.

60. Bhatia S, Jenney ME, Bogue MK, et al. The Minneapolis-Manchester Quality of Life instrument: reliability and validity of the Adolescent Form. J Clin Oncol. 2002;20(24):4692-4698.

61. Varni JW, Burwinkle TM, Katz ER, et al. The PedsQL in pediatric cancer: reliability and validity of the Pediatric Quality of Life Inventory Generic Core Scales, Multidimensional Fatigue Scale, and Cancer Module. Cancer. 2002;94(7):2090-2106.

62. Varni JW, Katz ER, Seid M, Quiggins DJL, Friedman-Bender A. The pediatric cancer quality of life inventory-32 (PCQL-32). Cancer. 1998;82(6):1184-1196. 
63. Seid M, Varni JW, Rode CA, Katz ER. The Pediatric Cancer Quality of Life Inventory: a modular approach to measuring health-related quality of life in children with cancer. Int J Cancer Suppl. 1999;12:71-76.

64. Eiser C, Havermans T, Craft A, Kernahan J. Development of a measure to assess the perceived illness experience after treatment for cancer. Arch Dis Child. 1995;72(4):302-307.

65. Eiser C, Kopel S, Cool P, Grimer R. The Perceived Illness Experience Scale (PIE): reliability and validity revisited. Child Care Health Dev. 1999;25(3):179-190.

66. Park CL, Wortmann JH, Hale-Smith AE, Cho D, Blank TO. Assessing quality of life in young adult cancer survivors: validity of the Survivorship-related Quality of Life Scale. American Psychological Association National Convention; August 2-5, 2012, 2012; Orlando, FL, USA.

67. Park CL, Wortmann JH, Hale-Smith AE, Cho D, Blank TO. Assessing quality of life in young adult cancer survivors: development of the survivorship-related Quality of Life Scale. 6th Biennial Cancer Survivorship Research Conference; June 14-16, 2012; Arlington, VA, USA.

68. Park EB, Emmons KM, Malloy NW, Seifer E. A qualitative exploration of health perceptions and behaviors among adult survivors of childhood cancers. J Cancer Educ. 2002;17(4):211-215.

69. Carlson LE, Speca M, Patel KD, Goodey E. Mindfulness-based stress reduction in relation to quality of life, mood, symptoms of stress and levels of cortisol, dehydroepiandrosterone sulfate (DHEAS) and melatonin in breast and prostate cancer outpatients. Psychoneuroendocrinology. 2004;29(4):448-474.

70. Cunningham AJ, Phillips C, Lockwood GA, Hedley DW, Edmonds CV. Association of involvement in psychological self-regulation with longer survival in patients with metastatic cancer: an exploratory study. $A d v$ Mind Body Med. 2000;16(4):276-287.

71. Cunningham AJ, Phillips C, Stephen J, et al. Fighting for life: a qualitative analysis of the process of psychotherapy-assisted self-help in patients with metastatic cancer. Integr Cancer Ther. 2002;1(2): 146-161.

72. Zabora J, BrintzenhofeSzoc K, Curbow B, Hooker C, Piantadosi S. The prevalence of psychological distress by cancer site. Psychooncology. 2001;10(1):19-28.

73. Bultz BD, Carlson LE. Emotional distress: the sixth vital sign in cancer care. J Clin Oncol. 2005;23(26):6440-6441.

74. Carlson LE, Bultz BD. Cancer distress screening. Needs, models, and methods. J Psychosom Res. 2003;55(5):403-409.

75. Thompson K, Palmer S, Dyson G. Adolescents and young adults: issues in transition from active therapy into follow-up care. Eur J Oncol Nurs. 2009;13(3):207-212.
76. Zebrack B, Mathews-Bradshaw B, Siegel S. Quality cancer care for adolescents and young adults: a position statement. J Clin Oncol. 2010;28(32):4862-4867.

77. Boehmer S, Luszczynska A. Two kinds of items in quality of life instruments: 'indicator and causal variables' in the EORTC qlq-c30. Qual Life Res. 2006;15(1):131-141.

78. Soliman H, Agresta SV. Current issues in adolescent and young adult cancer survivorship. Cancer Control. 2008;15(1):55-62.

79. Smith AB, Selby PJ, Velikova G, et al. Factor analysis of the Hospital Anxiety and Depression Scale from a large cancer population. Psychology and Psychotherapy: Theory, Research and Practice. 2002; 75:165-176.

80. Lovibond PF, Lovibond SH. The structure of negative emotional states: comparison of the Depression Anxiety Stress Scales (DASS) with the Beck Depression and Anxiety Inventories. Behav Res Ther. 1995;33(3):335-343.

81. Andrews G, Slade T. Interpreting scores on the Kessler Psychological Distress Scale (K10). Aust N Z J Public Health. 2001;25(6):494-497.

82. Greco LA, Baer RA, Smith GT. Assessing mindfulness in children and adolescents: development and validation of the Child and Adolescent Mindfulness Measure (CAMM). Psychol Assess. 2011;23(3): 606-614.

83. Greco LA, Lambert W, Baer RA. Psychological inflexibility in childhood and adolescence: development and evaluation of the Avoidance and Fusion Questionnaire for Youth. Psychol Assess. 2008;20(2): 93-102.

84. Leavitt M, Martinson IM, Liu CY, et al. Common themes and ethnic differences in family caregiving the first year after diagnosis of childhood cancer: part II. J Pediatr Nurs. 1999;14(2):110-122.

85. Ramirez AJ, Bogdanovic MR, Jasovic GM. Psychosocial adjustment to cancer: cultural considerations. The European Journal of Psychiatry. 1991;5:9-18.

86. Giuliano AR, Mokuau N, Hughes C, et al. Participation of minorities in cancer research: the influence of structural, cultural, and linguistic factors. Ann Epidemiol. 2000;10(8):S22-S34.

87. Abbott J, Baumann U, Conway S, et al. Cross cultural differences in health related quality of life in adolescents with cystic fibrosis. Disabil Rehabil. 2001;23(18):837-844.

88. Klopovich PM, Clancy BJ. Sexuality and the adolescent with cancer. Semin Oncol Nurs. 1985;1(1):42-48.

89. Mattsson E, Ringnér A, Ljungman G, von Essen L. Positive and negative consequences with regard to cancer during adolescence. Experiences two years after diagnosis. Psychooncology. 2007;16(11):1003-1009.
Clinical Oncology in Adolescents and Young Adults

\section{Publish your work in this journal}

Clinical Oncology in Adolescents and Young Adults is an international, peer-reviewed, open access journal publishing original research, reports, editorials, reviews and commentaries on all aspects of epidemiology, diagnosis and treatment of cancers in adolescents and young adults. The manuscript management system is completely

\section{Dovepress}

online and includes a very quick and fair peer-review system. Visit http://www.dovepress.com/testimonials.php to read real quotes from published authors. 